\title{
White Paper: Curriculum in Interventional Radiology White Paper: Curriculum Interventionelle Radiologie
}

Authors

Andreas H. Mahnken ${ }^{1}$, Arno Bücker ${ }^{2}$, Christian Hohl ${ }^{3}$, Ansgar Berlis ${ }^{4}$

Affiliations

1 Diagnostic \& Interventional Radiology, Philipps-University Marburg, Germany

2 Clinic of Diagnostic and Interventional Radiology, Saarland University Medical Center, Homburg, Germany

3 Radiologie, St. Marien-Krankenhaus Siegen, Germany

4 Neuroradiologie, Neurozentrum, Freiburg, Germany

Key words

education, interventional radiology, curriculum

received 25.01.2017

accepted 22.02.2017

Bibliography

DOI http://dx.doi.org/10.1055/s-0043-104773

Published online: 2017 | Fortschr Röntgenstr 2017; 189: 309-311

(c) Georg Thieme Verlag KG Stuttgart · New York

ISSN 1438-9029

\section{Correspondence}

Prof. Andreas H. Mahnken

Diagnostic \& Interventional Radiology, Philipps-University Marburg

Baldingerstraße

35043 Marburg

Germany

Tel.: ++49/6421/5866230

mahnken@med.uni-marburg.de

\section{ABSTRACT}

Purpose Scope and clinical importance of interventional radiology markedly evolved over the last decades. Consequently it was acknowledged as independent subspecialty by the „European Union of Medical Specialists“ (UEMS). Based on radiological imaging techniques Interventional Radiology is an integral part of Radiology.

Materials und Methods In 2009 the German Society for Interventional Radiology and minimally-invasive therapy (DeGIR) developed a structured training in Interventional Radiology. In cooperation with the German Society of Neuroradiology (DGNR) this training was extended to also cover Interventional Neuroradiology in 2012. Tailored for this training in Interventional Radiology a structured curriculum was developed, covering the scope of this modular training.

Results The curriculum is based on the DeGIR/DGNR modular training concept in Interventional Radiology. There is also an European Curriculum and Syllabus for Interventional Radiology developed by the "Cardi- ovascular and Interventional Radiological Society of Europe" (CIRSE). The presented curriculum in Interventional Radiology is designed to provide a uniform base for the training in Interventional Radiology in Germany, based on the competencies obtained during residency.

Conclusion This curriculum can be used as a basis for training in Interventional Radiology by all training sites.

\section{Key Points:}

- Interventional Radiology is an integral part of clinical radiology

- The German Society for Interventional Radiology and minimallyinvasivetherapy (DeGIR) developed a curriculum in Interventional Radiology

- This curriculum is an integrative basis for the training in interventional

\section{Citation Format}

- Mahnken AH, Bücker A, Hohl C et al. White Paper: Curriculum in Interventional Radiology. Fortschr Röntgenstr 2017; 189: 309-311

\section{ZUSAMMENFASSUNG}

Ziel Die interventionelle Radiologie hat in den letzten Jahrzehnten an Umfang und Bedeutung gewonnen und wurde mittlerweile durch die „European Union of Medical Specialists“ (UEMS) als eigenständige Subspezialität anerkannt. Sie ist integraler Bestandteil der Radiologie und basiert auf radiologischen Bildgebungs- und bildgestützten Behandlungstechniken.

Material und Methoden Die Deutsche Gesellschaft für Interventionelle Radiologie und minimalinvasive Therapie (DeGIR) hat 2009 eine zertifizierte Weiterbildung für die Fortbildung in interventioneller Radiologie entwickelt. Im Jahr 2012 wurde dies in Zusammenarbeit mit der Deutschen Gesellschaft für Neuroradiologie (DGNR) auf das neurointerventionelle Spektrum ausgedehnt. Zugeschnitten auf diese Weiterbildung wurde nun ein Curriculum entwickelt, das die Anforderungen an diese modulare Weiterbildung strukturiert abbildet.

Ergebnisse Das Curriculum basiert auf dem DeGIR/DGNR-Modul- und Stufenkonzept. Auch auf Ebene der europäischen Fachgesellschaft "Cardiovascular and Interventional Radiological Society of Europe (CIRSE) gibt es ein Curriculum für die Fortbildung in interventioneller Radiologie („European Curriculum and Syllabus for Interventional Radiology“). Mit dem vorliegenden Curriculum soll eine einheitliche Basis für die Fortbildung zum interventionellen Radiologen in Deutschland gelegt werden, wobei es auf den in der Facharztweiterbildung zum Radiologen erworbenen Kenntnissen aufbaut.

Schlussfolgerung Dieses Curriculum der DeGIR kann als Grundlage für die Fortbildung an den einzelnen zertifizierten Ausbildungsstätten für interventionelle Radiologie verwendet werden. 


\section{Introduction}

Interventional radiology is an integral part of clinical radiology. Image-guided diagnostic and therapeutic procedures are at the core of this specialty. In addition to minimally invasive interventions, important elements of interventional radiology include pathology, various imaging techniques and particularly patient management [1]. There are significant overlaps with other clinical specializations that use image guidance techniques and catheter techniques primarily developed in radiology. Since image guidance based on radiological techniques is a core element of interventional radiology, fundamental knowledge of radiation biology and radiation protection is essential for interventional radiologists $[2,3]$.

Interventional radiology has developed rapidly over the last decades. The type of procedures, the increasing number of procedures and in particular the growing complexity of procedures have resulted in special requirements regarding the training of radiologists [2]. In addition to knowledge of the type, implementation, and results of interventional-radiological procedures, the manual aspect must also be taken into consideration. In light of the increasing complexity and the necessary level of specialization, interventional radiology recently became established as its own specialty in the USA, for example. In 2015, a residency program for interventional radiology was introduced by the "Accreditation Council for Graduate Medical Education” [4]. In Europe, the European Union of Medical Specialists (UEMS) recognized interventional radiology as an independent subspecialty of radiology in 2009 [5]. A particular objective of the "UEMS Specialist Division - Interventional Radiology" has been to establish standards for the education and training of interventional radiologists. CIRSE created an interventional radiology curriculum ending with a certification examination (European Board of Interventional Radiology - EBIR) [2, 6].

In Germany, interventional radiology has grown since 2008 from a working group within the German Roentgen Society (DRG) into the German Society for Interventional Radiology and Minimally Invasive Therapy (DeGIR). As an affiliated society, the DeGIR is firmly rooted in the DRG. Since specialist training in radiology already includes extensive requirements, the DeGIR does not see a need to create a separate core area. Analogously to the "UEMS Specialist Division - Interventional Radiology", a main goal of the DeGIR is to establish training standards for interventional radiology including highly specialized interventional procedures. This objective is pursued in close cooperation with the German Society for Neuroradiology (DGNR). The aim is to establish a uniform standard regarding the knowledge and skills required by interventional radiologists. It must be taken into consideration that the broad spectrum of interventional radiology includes many areas, such as revascularization procedures and oncological and neuroradiological interventions.

To this end, a two-level modular concept was developed based on the qualification initiative of the DeGIR. Using two levels and six modules, this concept represents the requirements for interventional radiologists and neuroradiologists ( $\bullet$ Table 1 ). Training is concluded with an examination [7].
- Table 1 Modules of the DeGIR/DGNR module and level concept.

\begin{tabular}{|l|l|}
\hline name & short description of the module content \\
\hline module A & $\begin{array}{l}\text { vessel-opening procedures, incl. lysis, PTA, stent, } \\
\text { endoprosthesis, thrombectomy, etc. }\end{array}$ \\
\hline module B & $\begin{array}{l}\text { vessel-closing procedures, incl. coils, liquid embolic } \\
\text { agents, particles, plugs, etc. }\end{array}$ \\
\hline module C & $\begin{array}{l}\text { diagnostic punctures, drainages, PTCD, bile ducts, TIPPS, } \\
\text { gastrostomy, port, etc. }\end{array}$ \\
\hline module D & $\begin{array}{l}\text { oncological procedures, incl. TACE or other tumor- } \\
\text { specific embolization procedures, ablation, percutaneous } \\
\text { tumor treatments }\end{array}$ \\
\hline module E & $\begin{array}{l}\text { vessel-opening neuro-interventions (PTA/stent of the } \\
\text { extracranial supraaortic arteries, PTA/stent of the intra- } \\
\text { cranial arteries, mechanical recanalization in stroke, } \\
\text { local lysis and spasmolysis in stroke) }\end{array}$ \\
\hline $\begin{array}{l}\text { neurovascular embolization treatments (embolization } \\
\text { and comparable procedures for intracranial aneurysms, } \\
\text { embolization of intracranial and spinal vascular malfor- } \\
\text { mations, other embolization procedures in cerebral ves- } \\
\text { sels, vessels supplying the brain and vessels supplying } \\
\text { the spinal cord) }\end{array}$ \\
\hline module F
\end{tabular}

The creation of this modular program was accompanied by the establishment of a network of training and internship centers. In the future, specialist training in interventional radiology is to be performed at certified training centers. These centers must be able to show a documented minimum number of procedures for the individual modules via the DeGIR quality assurance software. Moreover, a trainer with corresponding proof of the highest certification of the DeGIR must be present.

\section{Interventional radiology curriculum}

A standardized curriculum is needed to define uniform training content in Germany. With the "European Curriculum and Syllabus for Interventional Radiology", the "Cardiovascular and Interventional Radiological Society of Europe" (CIRSE) created a basis for standardized training in Europe in 2013 [10]. However, the structure and parts of the content do not correspond with the requirements and reality of training in the field of interventional radiology in Germany. Therefore, the DeGIR created an interventional radiology curriculum that complements the DeGIR/DGNR module and level concept. This curriculum builds on and further develops diagnostic and interventional radiology/neuroradiology content already taught during specialist training in radiology. This curricular catalog presents the possible scope of each module without weighting the individual topics.

The DeGIR curriculum for interventional radiology is comprised of cross-module topics ( $\triangleright$ Table 2 ) and specific modulerelated requirements. The latter are consistently structured for the different modules according to technique, material, image guidance, and procedural considerations as illustrated in the example of $\downarrow$ Table 3 on the basis of catheter angiography as part of module $A$. 
- Table 2 Cross-module topics of the DeGIR curriculum in interventional radiology.

\section{General knowledge and radiation protection}

\section{general knowledge}

- anatomy of the arteries and veins, vascular territories (functional brain areas)

- basic principles of hemodynamics and vascular physiology

- basic principles of analgesia and sedation

- basic principles of blood coagulation and the pharmacology of anticoagulants and their antagonism

- basic principles of pathology, pathophysiology, and clinical assessment of module-typical diseases

- pharmacology, risks and complication profile of contrast agents

- alternative and complementary diagnostic and treatment methods (value of noninvasive diagnostic methods, standard vascular surgery treatments, hybrid procedures, conservative therapy approaches, etc.)

- basic principles of peri-interventional monitoring

- basic principles of interdisciplinary indication determination and collaboration

- basic principles of quality assurance (DeGIR QA, legally required QA)

radiation protection in interventional-radiological procedures

- active radiation protection (pulsed fluoroscopy, last image hold, recording of fluoroscopy series, collimation, exposure geometry)

- passive radiation protection (protective clothing, leaded glass goggles, radiation protection attached to equipment, radiation protection shield), special considerations in $\mathrm{CT}$ interventions with high kV values

- dosimetry, dose structure report (DSR).

- Table 3 Example of a module-related theme of DeGIR Curiculums Interventional Radiology.

Aortic, visceral, and peripheral catheter angiography

\begin{tabular}{|c|c|}
\hline Technique & $\begin{array}{l}\text { Cross-over technique, selective and } \\
\text { superselective catheter techniques }\end{array}$ \\
\hline Materials & $\begin{array}{l}\text { Plain and selective catheters, manual } \\
\text { and automated contrast injection. Ad- } \\
\text { vantages and disadvantages of different } \\
\text { contrast agents (e. g., iodine-containing } \\
\text { contrast agent, CO2, etc.), medication } \\
\text { (butylscopolamine, vasodilators, etc.) }\end{array}$ \\
\hline Guidance & Fluoroscopy (pulsed, high-dose), DSA \\
\hline Special considerations & $\begin{array}{l}\text { Patients with renal insufficiency, intole- } \\
\text { rance to contrast agents }\end{array}$ \\
\hline
\end{tabular}

This curriculum is a living document and should be modified and supplemented according to developments in interventional radiology. This curriculum makes it possible for the first time in Germany to teach interventional radiology and neuroradiology in a structured manner. The curriculum can be used as a basis for the individual structuring of training at the training centers for interventional radiology and/or neuroradiology. It also provides the basis for the level-2 examinations "specialization in interventional radiology and/or neuroradiology". In the future, holders of level-2 certificates will be able to refer to themselves as "certified interventional radiologists of DeGIR (level 2)" or "certified interventional radiologists/neuroradiologists of DeGIR/DGNR (level 2)". Depending on the modules in which level-2 certification is acquired, various additional qualifications such as vascular medicine or interventional oncology are possible.

\section{Conflict of Interest}

The authors declare that they have no conflict of interest.

\section{References}

[1] Goltz JP, Janssen H, Petritsch B et al. Launching a Permanent Out-ofHour Interventional Radiology Service: Single-Center Experience from a German University Hospital. Fortschr Röntgenstr 2014; 186: 136-141

[2] Tsetis D, Uberoi R, Fanelli F et al. The Provision of Interventional Radiology Services in Europe: CIRSE Recommendations. Cardiovasc Intervent Radiol 2016; 39: 500 - 506

[3] Kamusella P, Wissgott C, Wiggermann P et al. Reduction of Radiation Exposure for the Examiner in Angiography using a Direct Dosimeter. Fortschr Röntgenstr 2013; 185: $241-245$

[4] Siragusa DA, Cardella JF, Hieb RA et al. Requirements for training in interventional radiology. J Vasc Interv Radiol 2013; 24: 1609-1612

[5] https://www.uems.eu/about-us/medical-specialties; last accessed January 24, 2017

[6] Lee M], Belli AM, Brountzos E et al. Specialty status for interventional radiology: the time is now. Cardiovasc Intervent Radiol 2014; 37: 862

[7] http://www.degir.de/de-DE/2525/degir-dgnr-zertifizierung; last accessed January 24, 2017

[8] Heuser L, Arnold CN, Morhard D et al. DeGIR-Qualitätsreport 2011 Bericht über die Behandlungsqualität minimalinvasiver Methoden. Fortschr Röntgenstr 2012; 184: 570 - 576

[9] Heuser L], Arnold CN, Morhard D et al. Qualitätsreport 2011: Bericht über die Behandlungsqualität minimalinvasiver Methoden - Teil 2 Interventionelle Therapie (EVAR) von Aortenaneurysmen. Fortschr Röntgenstr 2013; 185: 709-719

[10] http://www.cirse.org/?pid=378; last accessed January 24, 2017 